\title{
Polygenic scores for schizophrenia and general cognitive ability: associations with six cognitive domains, premorbid intelligence, and cognitive composite score in individuals with a psychotic disorder and in healthy controls
}

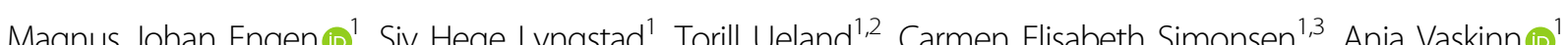

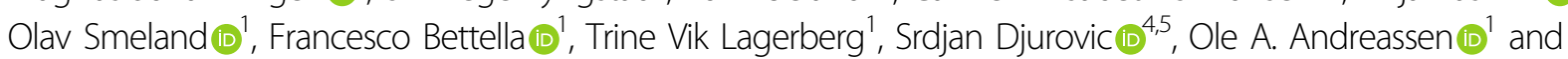
Ingrid Melle (1)

\begin{abstract}
Cognitive impairments are considered core features in schizophrenia and other psychotic disorders. Cognitive impairments are, to a lesser degree, also documented in healthy first-degree relatives. Although recent studies have shown (negative) genetic correlations between schizophrenia and general cognitive ability, the association between polygenic risk for schizophrenia and individual cognitive phenotypes remains unclear. We here investigated the association between a polygenic score for schizophrenia $\left(S \mathrm{CZ}_{\mathrm{PGS}}\right.$ ) and six well-defined cognitive domains, in addition to a composite measure of cognitive ability and a measure of premorbid intellectual ability in 731 participants with a psychotic disorder and 851 healthy controls. We also investigated the association between a PGS for general cognitive ability (COGPGS) and the same cognitive domains in the same sample. We found no significant associations between the $S_{C} Z_{P G S}$ and any cognitive phenotypes, in either patients with a psychotic disorder or healthy controls. For COGPGS we observed stronger associations with cognitive phenotypes in healthy controls than in participants with psychotic disorders. In healthy controls, the association between COGPGS (at the $p$ value threshold of $\geq 0.01$ ) and working memory remained significant after Bonferroni correction $\left(\beta=0.12, p=8.6 \times 10^{-5}\right)$. Altogether, the lack of associations between $\mathrm{SCZ}_{\mathrm{PGS}}$ and $\mathrm{COG}_{\mathrm{PGS}}$ with cognitive performance in participants with psychotic disorders suggests that either environmental factors or unassessed genetic factors play a role in the development of cognitive impairments in psychotic disorders. Working memory should be further studied as an important cognitive phenotype.
\end{abstract}

\section{Introduction}

Cognitive impairment is a core feature of schizophrenia and other psychotic disorders ${ }^{1}$. Impairments are most

\footnotetext{
Correspondence: Magnus Johan Engen (m.j.engen@psykologi.uio.no) ${ }^{1}$ NORMENT, Division of Mental Health and Addiction, Oslo University Hospital \& Institute of Clinical Medicine, University of Oslo, Oslo, Norway

${ }^{2}$ Department of Psychology, Faculty of Social Sciences, University of Oslo, Oslo, Norway

Full list of author information is available at the end of the article
}

prominent in schizophrenia ${ }^{2-5}$, but also frequent in bipolar disorders ${ }^{3,6-8}$. The genetic contribution to the risk of developing a psychotic disorder is high, with heritability estimates for schizophrenia and bipolar disorder ranging from 0.6 to $0.8^{9}$. Both disorders show high degrees of polygenicity and have a considerable genetic overlap ${ }^{10}$. The genetic contribution to cognitive abilities is also substantial with a heritability estimate of $0.5^{11}$. It is now known that the phenotypic link between cognitive 
domains and a number of different psychiatric diagnoses is at least partly explained common genetic variants ${ }^{12}$. Mild cognitive impairments have been found in firstdegree relatives of probands with both schizophrenia and bipolar disorder ${ }^{13,14}$, and a recent study estimated that as much as one-third of the genetic risk for schizophrenia could be mediated through cognition-relevant pathways ${ }^{15}$. Understanding the genetic underpinnings of cognitive phenotypes in psychotic disorders could be key in understanding the etiology of these disorders. The considerable negative impact of cognitive impairment on functional outcome and our current lack of efficient treatment strategies emphasize the importance of identifying the underlying mechanisms ${ }^{16,17}$.

The wealth of data uncovered by large-scale genomewide association studies (GWAS) offers promising research opportunities for exploring the genetic architecture of psychotic disorders. The Psychiatric Genomics Consortium (PGC) identified 108 separate genetic loci statistically associated with schizophrenia risk after correction for multiple testing, which accounted for $3.4 \%$ of the variation in liability to schizophrenia ${ }^{18}$. These loci meet the high standard set for "true-positive" hits, but applying more liberal $p$ value thresholds (PTs) reveals that the actual polygenic architecture of schizophrenia comprises thousands of genetic variants, each with very small effects that in aggregate explain a third to a half of the heritability of the disorder ${ }^{18}$. This is the basis for the calculation of polygenic scores (PGSs). Here common allele associations from a GWAS discovery sample are used to calculate an aggregate PGS as the cumulative effect sizes that differ between cases and controls. PGSs can be created for any investigated phenotype and can be used to explore the associations between the polygenic basis of this phenotype and other individual characteristics.

How the PGS for schizophrenia $\left(\mathrm{PGS}_{\mathrm{SCZ}}\right)$ is related to cognition has been studied in both clinical samples and in the general population. Results from studies of clinical samples have been mixed: While one study found strong negative associations between $\mathrm{PGS}_{\mathrm{SCZ}}$ and seven cognitive domains ${ }^{19}$, other studies have reported a lack of significant associations ${ }^{20-23}$. Two studies have in addition found $\mathrm{PGS}_{\mathrm{SCZ}}$ to be negatively associated with general cognitive ability in healthy controls but not in patients $^{24,25}$. A plausible explanation for the latter findings is that the effect of the association between PGS $_{\mathrm{SCZ}}$ and cognition is overshadowed by non-genetic illness factors in patients, creating stronger associations between genetic and cognitive measures in the healthy control group than in the patient group. In line with this, one of these studies ${ }^{25}$ found that higher PGS for intelligence was associated with better general cognitive ability in both patients and healthy controls, but with a stronger effect in healthy controls. However, the study did not explore the effect on specific cognitive domains.

As there is no consensus on the operationalization of cognitive domains, studies vary in their selection of cognitive tests. In genetic studies, general cognitive ability is frequently operationalized as a composite score of available tests referred to as Spearman's $g$, a term for the latent variable thought to underlie all cognitive domains ${ }^{26}$. Because $g$ is confirmed as a valid construct ${ }^{26,27}$ with high heritability $^{26,28}$, and allows for the combination of unrelated cognitive tests to increase sample size and statistical power, its frequent use in genetic studies is unsurprising. However, substantial and independent genetic effects on specific cognitive domains have also been demonstrated ${ }^{27}$. The variation in both sample sizes and cognitive phenotypes used in studies of $\mathrm{PGS}_{\mathrm{SCZ}}$ makes interpretation of results challenging ${ }^{29}$, and at present only one study has investigated how the $\mathrm{PGS}_{\mathrm{SCZ}}$ is associated with a broader selection of cognitive phenotypes known to be influenced by psychosis in both individuals with psychosis and healthy controls ${ }^{19}$.

In summary: While a genetic basis for cognitive impairment in psychotic disorders appears established $^{15,30,31}$, the nature of this association is complex and its true extent remains unclear. Twin studies indicate that the shared genetic basis for schizophrenia and cognition is substantial ${ }^{32,33}$. However, a population-based twin study with measures of premorbid cognitive ability found only $7 \%$ shared genetic variance between psychosis and cognition $^{34}$. In addition, while the association between $g$ and cognitive domains are considerable, they are not identical and probably have different genetic architectures with different associations to the genetic basis of psychotic disorders ${ }^{35}$.

To increase our understanding of the genetic relationship between psychotic disorders and cognitive function, we here investigated the association between $\mathrm{PGS}_{\mathrm{SCZ}}$ and a well-defined set of cognitive domains in a large sample of participants with a psychotic disorder and healthy controls. In addition, we explored the associations between the PGSs for general cognition $\left(\mathrm{PGS}_{\mathrm{COG}}\right.$ ) based on a GWAS with stringent inclusion criteria, and the same set of cognitive domains in the same group of participants. Our hypothesis was that we would find negative associations between the $\mathrm{PGS}_{\mathrm{SCZ}}$ and cognitive domain scores and positive associations between and $\mathrm{PGS}_{\mathrm{COG}}$ and cognitive domain scores in both groups, but that the effect size of the associations would be higher in healthy controls. We also included an estimate of premorbid intelligence, and hypothesized less differences between participants with psychosis and healthy controls for this measure. 


\section{Methods}

\section{Participants, clinical measures, and study design}

The current study was based on the inclusion criteria for the thematically organized psychosis study. All recruited participants were between 18 and 65 years. For the research questions in the current study, we included participants with declared European ancestry who had participated in cognitive assessment and met the criteria for a psychotic disorder. Psychotic bipolar disorder was included based on genetic overlap with schizophrenia and the phenotypic similarity with regards to cognitive impairment. The sample included participants with schizophrenia spectrum disorders $(N=522)$ (schizophrenia $n=294$, schizophreniform disorder $n=28$, schizoaffective disorder $n=72$, and psychosis not otherwise specified $n=128)$ and psychotic bipolar spectrum disorders $(n=$ 209) (bipolar I disorder $n=170$, bipolar II disorder $n=$ 28 , bipolar disorder not otherwise specified $n=11$ ), resulting in a clinical sample of 731 psychosis spectrum participants. Clinical interviews for DSM-IV (Diagnostic and Statistical Manual of Mental Disorders, fourth edition) diagnoses in patients ${ }^{36}$ were performed by psychologists, psychiatrists, or medical doctors. Healthy controls $(n=851)$ were randomly selected from the population registry in the same catchment area. The healthy controls were screened with the Primary Care Evaluation of Mental Disorders (PRIME-MD) ${ }^{37}$ and a family history interview, performed by individuals with a master's degree in psychology or neuroscience. Controls were not included if they had a personal or family (first-degree) history of a psychotic disorder. Both psychosis spectrum participants and healthy controls were excluded if they did not speak a Scandinavian language, had a history of serious head injury, suffered from a known medical or neurological condition interfering with brain functioning, or intelligence quotient $<70$. Demographic and clinical characteristics are presented in Table 1.

Table 1 Demographic and clinical variables.

\begin{tabular}{lll}
\hline & $\begin{array}{l}\text { Healthy } \\
\text { controls }\end{array}$ & $\begin{array}{l}\text { Individuals with } \\
\text { psychosis }\end{array}$ \\
\hline N Total (\% females) & $851(47)$ & $731(48)$ \\
Mean age (SD) & $32.7(9.0)$ & $31.3(10.8)$ \\
Education years (SD) & $14.4(2.2)$ & $12.8(2.6)$ \\
AP medication ${ }^{\mathrm{a}}$ (SD) & & $1.0(0.7)$ \\
Duration of illness $^{\mathrm{b}}$ (SD) & & $6.3(7.4)$ \\
\hline
\end{tabular}

$S D$ standard deviation, $A P$ antipsychotic.

${ }^{\text {aD }}$ Daily antipsychotic dosage relative to the average recommended daily dosage. One hundred and seventy-five individuals with missing data.

${ }^{b}$ Years from first episode with psychosis or mania. Twenty-seven individuals with missing data.
The Positive and Negative Syndrome Scale ${ }^{38}$ Wallwork's five factor model $^{39}$ was used to measure symptoms. The Alcohol Use Disorder Identification Test and the Drug Use Disorder Identification Test ${ }^{40}$ were used to assess alcohol use and drug use, respectively. Duration of illness was based on information from the severe combined immunodeficiency interview, and was calculated as the time in years from the first episode of psychosis or mania. Use of antipsychotic medication was recoded into defined daily dosages ${ }^{41}$.

All participants in the cognitive assessments were included to increase statistical power. However, some participants were lacking scores for one or more subtests, precluding the calculation of a cognitive composite score for these individuals. Therefore, the sample varies from $n=726$ for verbal learning to $n=533$ for the cognitive composite score. Mean scores and Ns for participants with psychosis and healthy controls for all tests and domains are presented in Table 2. Written informed consent was obtained from all participants.

\section{Genetic analyses}

DNA samples obtained from blood or saliva were sent for genotyping in six separate batches between the years of 2014 and 2017 at deCODE Genetics (Reykjavik, Iceland). The samples were analyzed using Illumina Human OmniExpress12 (first four batches), Infinium OmniExpress24 (fifth batch), and Illumina Global Screening Array (sixth batch) platforms, respectively. Identical but independent procedures were followed for sample batches genotyped on different platforms. PLINK version $1.9^{42}$ was deployed to perform the necessary pre-imputation quality control. This involved removal of any samples with possible contamination (heterozygosity more than five standard deviations above the mean) or too low coverage $((<80 \%)$ to enable its detection) and any variants with genotyping rate lower than 95\%, Hardy-Weinberg disequilibrium test $p$ value lower than $10^{-4}$, and high rate of Mendel errors (https://www.cog-genomics.org/plink/ 1.9/basic_stats\#Mendel) in eventual trios or significant (false discovery rate $<0.5$ ) batch effects (in case multiple batches were being processed simultaneously). The quality-controlled genotypes were phased using Eagle $^{43}$, and missing variants were imputed with $\mathrm{MaCH}^{44,45}$ using version 1.1 of the trans-ethnic reference sample put together by the haplotype reference consortium (HRC) ${ }^{46}$. High-quality variant sets from the quality control procedure (see Supplementary information) were selected to impute each individual's sex and compute each individual's genetic principal components (PCs). The latter consisted of the individual's components along the 20 first eigenvectors of the pairwise genetic covariance matrix of a sub-sample of unrelated individuals from the reference (HRC) panel. Following the quality control and 
Table 2 Cognitive assessment scores.

\begin{tabular}{|c|c|c|c|c|}
\hline & \multicolumn{2}{|c|}{ Psychosis } & \multicolumn{2}{|c|}{ Controls } \\
\hline & $N$ & Mean \pm SD & $N$ & Mean \pm SD \\
\hline \multicolumn{5}{|l|}{ Cognitive domain } \\
\hline Verbal learning & 726 & $-0.75 \pm 1.27$ & 850 & $0.00 \pm 1.00$ \\
\hline CVLT-II verbal learning ${ }^{a}$ & 537 & $50.18 \pm 11.79$ & 380 & $57.14 \pm 9.73$ \\
\hline HVLT verbal learning ${ }^{b}$ & 189 & $24.87 \pm 5.58$ & 472 & $28.23 \pm 3.94$ \\
\hline Verbal memory & 685 & $-0.69 \pm 1.31$ & 850 & $0.00 \pm 1.00$ \\
\hline CVLT-II delayed free recall ${ }^{a}$ & 535 & $11.42 \pm 3.37$ & 380 & $13.21 \pm 2.69$ \\
\hline HVLT delayed free recall ${ }^{b}$ & 150 & $8.85 \pm 2.60$ & 472 & $10.20 \pm 1.73$ \\
\hline Processing speed & 679 & $-0.96 \pm 1.13$ & 845 & $0.00 \pm 0.78$ \\
\hline WAIS digit-symbol coding ${ }^{a}$ & 536 & $62.03 \pm 15.56$ & 380 & $77.46 \pm 13.23$ \\
\hline BACS digit-symbol coding ${ }^{b}$ & 189 & $47.43 \pm 10.90$ & 472 & $58.31 \pm 9.15$ \\
\hline D-KEFS color naming ${ }^{* d}$ & 682 & $33.23 \pm 7.16$ & 847 & $28.16 \pm 4.64$ \\
\hline D-KEFS word reading ${ }^{* d}$ & 682 & $23.56 \pm 5.02$ & 846 & $21.25 \pm 3.71$ \\
\hline \multicolumn{5}{|l|}{ Executive functions } \\
\hline Working memory & 564 & $-0.60 \pm 0.88$ & 812 & $0.00 \pm 0.88$ \\
\hline WAIS letter-number sequencing ${ }^{a}$ & 461 & $9.47 \pm 2.38$ & 366 & $11.51 \pm 2.56$ \\
\hline MCCB letter-number sequencing ${ }^{b}$ & 184 & $13.58 \pm 3.17$ & 472 & $15.17 \pm 3.03$ \\
\hline WAIS digit span ${ }^{d}$ & 642 & $14.74 \pm 3.64$ & 827 & $16.44 \pm 3.71$ \\
\hline Attentional control & 678 & $-0.92 \pm 1.47$ & 824 & $0.00 \pm 0.86$ \\
\hline D-KEFS inhibition*,d & 682 & $59.00 \pm 16.51$ & 845 & $48.55 \pm 9.79$ \\
\hline D-KEFS inhibition-switching ${ }^{*, d}$ & 678 & $64.26 \pm 17.76$ & 825 & $55.13 \pm 11.67$ \\
\hline Category fluency & 693 & $-1.06 \pm 1.20$ & 850 & $0.00 \pm 0.99$ \\
\hline D-KEFS category fluency ${ }^{a}$ & 505 & $40.58 \pm 9.65$ & 381 & $49.25 \pm 7.85$ \\
\hline MCCB category fluency ${ }^{b}$ & 188 & $23.03 \pm 6.44$ & 470 & $28.46 \pm 5.87$ \\
\hline \multicolumn{5}{|l|}{ General cognitive ability } \\
\hline $\mathbf{N A R T}^{c, d}$ & 635 & $-0.47 \pm 1.19$ & 825 & $0.00 \pm 1.00$ \\
\hline Cognitive composite score & 533 & $-0.81 \pm 0.89$ & 802 & $0.00 \pm 0.61$ \\
\hline
\end{tabular}

All domains in bold are transformed into $z$-scores using the healthy control mean and standard deviation. The cognitive composite score is averaged over all domains except NART, which is an estimate of premorbid intellectual functioning.

*Scores reversed when transformed into $z$-scores to make higher values reflect better performance.

${ }^{\text {a }}$ Test from Battery 1.

best from Battery 2

'Test included in both assessment batteries.

imputation procedure, variants with information score $<0.8$ or minor allele frequency $<0.01$ were removed. In addition, individual genotypes imputed with $<75 \%$ confidence were set to missing, and the remaining ones were converted to best guess hard allelic dosages. Finally, individuals with imputation rate $<95 \%$ and an up to thirddegree relative with better imputation rate or differing annotated and imputed sex were excluded. For more detailed descriptions of the quality control and imputation procedures, refer to the Supplementary information.

\section{Polygenic scores}

The $\mathrm{SCZ}_{\mathrm{PGS}}$ were computed following the method described by Purcell et al. ${ }^{47}$. A meta-analysis covering all PGC sub-studies, except our sample, was performed to obtain risk allele effect sizes $[\ln (\mathrm{OR})]$ for all imputed variants. We then used PRSice ${ }^{48}$ to compute PGSs based on seven increasing PTs: $\mathrm{PT} \leq 5 \times 10^{-8}, \mathrm{PT} \leq 1 \times 10^{-5}$, $0.001,0.01,0.05,0.1$, and 0.5 . The SCZ discovery sample used for $\mathrm{SCZ}_{\mathrm{PGS}}$ is based on 36,989 participants with schizophrenia and 113,079 healthy controls. Participants 
come from a total of 49 case-control samples (46 European and 3 East Asian) and 3 family-based samples of European ancestry ${ }^{18}$.

The $\mathrm{PGS}_{\mathrm{COG}}$ were computed following the same procedure for seven increasing PTs: $\mathrm{PT} \leq 5 \times 10^{-8}$, $\mathrm{PT} \leq 1 \times$ $10^{-5}, 0.001,0.01,0.05,0.1$, and 0.5 , but using a metaanalysis of COGENT consortium sub-studies of general cognitive function ${ }^{49}$. This discovery sample consists of 35,298 individuals with European ancestry from 24 studies (mean age of 45.6 ( $\mathrm{SD} \pm 8.6$ ) $51.4 \%$ females). Most of these studies are of clinical populations (schizophrenia, Alzheimer's disease, cardiovascular disorders), but the healthy controls and participants in studies of the general population were also included.

\section{Measures of cognition}

All participants were assessed with a comprehensive cognitive assessment battery. At the start of the study, there was no international consensus regarding which tests to use and the study constructed a battery based on the knowledge about cognitive domains that were most affected by psychotic disorders ("battery 1"). With the development of the Measurement and Treatment Research to Improve Cognition in Schizophrenia (MATRICS) Consensus Cognitive Battery $(\mathrm{MCCB})^{50}$, the study decided to change to the MCCB ("battery 2"). Since both batteries focused on domains affected by psychotic disorders, they covered essentially the same areas, but used somewhat different specific tests. For the purpose of the study results from the two batteries were merged according to the following procedure ${ }^{51}$ : Cognitive test scores were first converted into $z$-scores based on the mean scores and standard SDs of the healthy control group. Tests were combined into the six specific cognitive domains based on their well-recognized relevance to cognitive impairment in psychotic disorders ${ }^{52}$, and included Attentional control, Category fluency, and Working memory (these three covering different aspects of Executive function) together with Processing speed, Verbal learning, and Verbal memory.

Attentional control comprised the inhibition and the inhibition/switching conditions of the Delis-Kaplan Executive Functions System (D-KEFS) Color-Word Interference task ${ }^{53}$; Category fluency was constructed merging the Category fluency test from D-KEFS ${ }^{53}$ and the Category fluency test from the $\mathrm{MCCB}^{50}$.

Processing speed was measured using the word reading and color naming conditions of the Color-Word Interference task from the D-KEFS ${ }^{53}$. In addition, the digit symbol tests from WAIS-III ${ }^{54}$ and BACS ${ }^{50,55}$ were included. Working memory consisted of the WAIS-III digit span, forward and backward, and the WAIS-III Letter-Number Sequencing test, merged with Letter-Number Span from the $\mathrm{MCCB}^{50}$. The Verbal learning and Verbal memory domains were constructed merging relevant conditions from the California Verbal Learning Test II and the Hopkins Verbal Learning Test-Revised, respectively ${ }^{50,56}$.

In addition to investigating these six domains separately, we also combined them into a Cognitive composite score as a measure of general cognitive ability. The cognitive composite score was constructed as the individual's mean $z$-score across the six domains. Finally, the National Adult Reading Test (NART) ${ }^{57}$ was included as an estimate of premorbid intelligence. The Norwegian NART is a valid measure of premorbid intelligence ${ }^{58}$.

\section{Statistical analyses}

We used SPSS version 25 for the analyses of the main research question. Means are reported for data with normal distribution and all test are two-tailed with $\alpha$ set to $p<0.05$. We started the analyses by selecting the relevant ancestry PCs to be included in the analyses, by bivariate association analyses between the 20 first ancestry PCs, and the ten $\mathrm{PGS}_{\mathrm{SCZ}} P_{\mathrm{T}}$ levels and case-control status, resulting in a list of $16 \mathrm{PCs}$ that were associated at the $p \leq 0.05$ level with any of these. The next step was to run ten separate logistic regressions (one for each $P_{\mathrm{T}}$ ) with case-control status as the dependent variable correcting for genotyping batch and the $16 \mathrm{PCs}$ to identify the $P_{\mathrm{T}}$ with the highest explained variance in case-control status. The $P_{\mathrm{T}}$ with the best fit based on the Akaike information criterion was $P_{\mathrm{T}} \leq 0.01$ (Fig. 1). $P_{\mathrm{T}} \leq 0.01$ was thus selected for testing for associations between $\mathrm{PGS}_{\mathrm{SCZ}}$ and the cognitive phenotypes in the subsequent analyses. For the PGS ${ }_{\mathrm{COG}}$, all available $P_{\mathrm{T}}$ levels were used in the main analyses.

The main analyses to investigate the association between $\mathrm{PGS}_{\mathrm{SCZ}} P_{\mathrm{T}} \leq 0.01$ and cognitive domains were multiple linear regression analyses, with the different cognitive phenotypes as dependent variables and the $\mathrm{PGS}_{\mathrm{SCZ}}$ as the independent variable introduced at the last step of the analysis after, controlling for age, sex, genotyping batch, and PCs. For this step, we selected PCs that had a bivariate association at the $p \leq 0.05$ level to either the $\mathrm{PGS}_{\mathrm{SCZ}} P_{\mathrm{T}} \leq 0.01$, the seven $\mathrm{PGS}_{\mathrm{COG}} P_{\mathrm{T}}$ levels or to any of the eight cognitive phenotypes, resulting in nine PCs. For the next research question, the multiple linear regression analyses were repeated, this time with the seven $\mathrm{PGS}_{\mathrm{COG}} P_{\mathrm{T}}$ levels as independents. All these analyses were done separately for psychosis participants and for healthy controls. The $\alpha$ level was set at 0.05 for all tests, with Bonferroni corrections. We adjusted for testing in two different populations for eight domains and one $\mathrm{PGS}_{\mathrm{SCZ}} P_{\mathrm{T}}$, yielding a corrected threshold of $p<0.003$ (16 tests) for $\mathrm{PGS}_{\mathrm{SCZ}}$ and likewise yielding a corrected threshold of $p<0.00045$ (112 tests) for the seven PGS ${ }_{\text {COG }}$ $P_{\mathrm{T}}$ levels. The difference in regression coefficients between cases and controls were tested using the method 


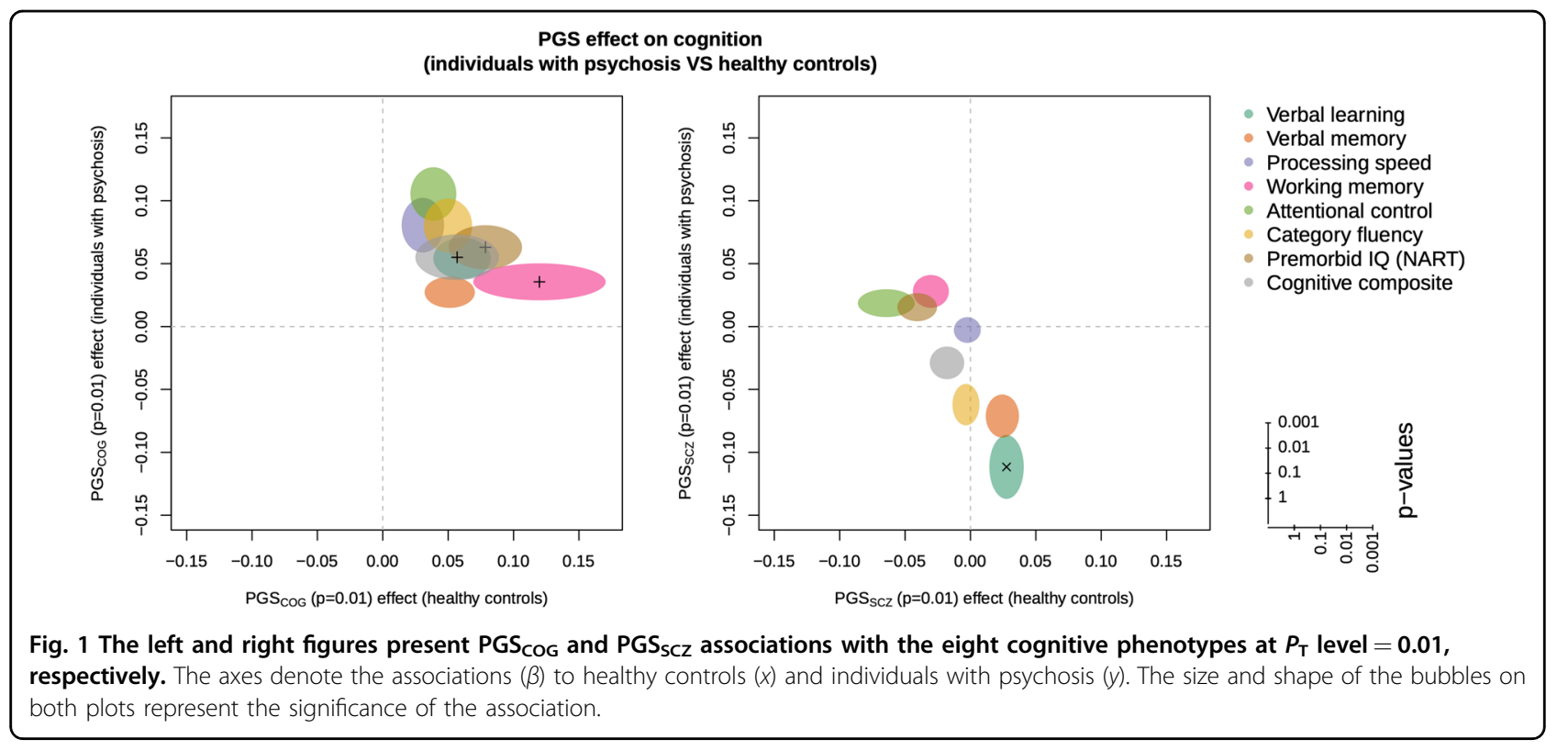

of Weaver and Wuensch, taking the sample size, the number of independent variables, and the standard error into account ${ }^{59}$. In the follow-up analyses, all regressions were repeated with only participants within the schizophrenia spectrum.

\section{Results}

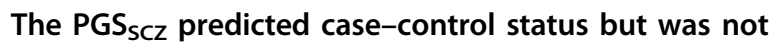
significantly associated with any cognitive domain

The associations between the $\mathrm{PGS}_{\mathrm{SCZ}}$ at $P_{\mathrm{T}} \leq 0.01$ and the cognitive phenotypes are displayed in Fig. 1. Except for a nominally significant association to verbal learning for individuals with psychosis $(\beta=-0.11, p=0.03)$, there were no significant associations between the $\mathrm{PGS}_{\mathrm{SCZ}}$ and the remaining cognitive phenotypes (numerical values for $\beta \mathrm{s}, 95 \%$ confidence intervals (CIs), and $p$ values for both participants with psychosis and healthy controls are presented in Supplementary Table S1). Repeating the analyses, including only participants within the schizophrenia spectrum, did not change the results.

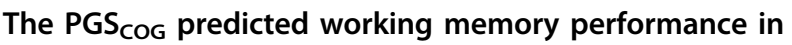 controls}

The analyses were repeated using the seven pre-selected PGS $_{\text {COG }} P_{\mathrm{T}}$ levels to evaluate how the genetic basis for general cognition was associated with the cognitive phenotypes. There were no associations between $\mathrm{PGS}_{\mathrm{COG}}$ and cognitive phenotypes that reached unadjusted levels of statistical significance across the seven $P_{\mathrm{T}} \mathrm{s}$ in participants with a psychotic disorder. In healthy controls, we observed the strongest associations between $\mathrm{PGS}_{\mathrm{COG}}$ and cognitive phenotypes at $P_{\mathrm{T}} \leq 0.01$, where nominally significant results were for the cognitive composite score $(\beta=0.06, p=0.01), \quad$ NART $(\beta=0.08, p=0.02)$, and working memory $(\beta=0.12, p=0.00009)$. Only the association with working memory reached the Bonferronicorrected level of significance. The adjusted $R^{2}$ for this model was 0.022 and the change in adjusted $R_{2}$ when entering the $\mathrm{PGS}_{\mathrm{COG}}$ at the last step was 0.017 . The association between cognitive phenotypes and $\mathrm{PGS}_{\mathrm{COG}}$ at $P_{\mathrm{T}} \leq 0.01$ are displayed for both psychosis participants and healthy controls in Fig. 1. (The numerical values for $\beta \mathrm{s}$, 95\% CIs, and $p$ values for both psychosis participants and healthy controls at all $P_{\mathrm{T}}$ s are presented as Supplementary material (Supplementary Table S2), while Supplementary Fig. S1 graphically shows the association at the six remaining $P_{\mathrm{T}}$ levels analyzed). Repeating the analyses including only patients within the schizophrenia spectrum did not change the results. The nominal $p$ value for the difference in regression coefficients between patients and controls for the analysis of working memory and PGS ${ }_{\text {COG }}$ at $P_{\mathrm{T}} \leq 0.01$ was 0.05 , below the level of statistical significance after correction for multiple testing.

\section{Symptoms and substance use did not moderate the association between $\mathrm{PGS}_{\mathrm{COG}}$ and cognitive phenotypes in the psychosis sample}

The analysis that yielded the strongest associations between the $\mathrm{PGS}_{\mathrm{COG}}$ and cognitive phenotypes in healthy controls at $P_{\mathrm{T}} \leq 0.01$ (working memory) were repeated in participants with a psychotic disorder, controlling for clinical characteristics that potentially could influence the associations (illness history, symptom and substance use scores, and antipsychotic medication use). Clinical variables with associations $p<0.1$ to the cognitive phenotypes (Supplementary Table S3) were entered in the analysis. 
This did not significantly alter the associations between PGS $_{\mathrm{COG}}$ and the cognitive phenotypes in the psychosis group. (The analysis for working memory and $\mathrm{PGS}_{\mathrm{COG}}$ at $P_{\mathrm{T}} \leq 0.01$ is shown in Supplementary Table S4.) Repeating the analyses including only patients within the schizophrenia spectrum did not affect the results.

\section{Discussion}

The current study is the largest to date investigating the association between a broad and well-defined set of cognitive domains and $\mathrm{PGS}_{\mathrm{SCZ}}$ and $\mathrm{PGS}_{\mathrm{COG}}$, in both healthy controls and participants with psychotic disorders. Overall, we did not find the hypothesized negative associations between $\mathrm{PGS}_{\mathrm{SCZ}}$ and cognitive phenotypes. However, we did observe a statistically (Bonferroni-corrected) significant association between $\mathrm{PGS}_{\mathrm{COG}}$ at $P_{\mathrm{T}} \leq$ 0.01 and working memory in healthy controls. This association in healthy controls was nominally stronger than in participants with psychotic disorders $(p=0.05)$.

A previous study, in a slightly smaller sample than the current, investigated the association between $\mathrm{PGS}_{\mathrm{SCZ}}$ and estimates of premorbid and current cognitive abilities in patients with psychosis and healthy controls ${ }^{24}$. They found that premorbid intelligence was not associated with $\mathrm{PGS}_{\mathrm{SCZ}}$, whereas better general cognitive ability was associated with lower $\mathrm{PGS}_{\mathrm{SCZ}}$ in healthy controls. The negative finding for premorbid cognitive abilities was replicated in a large population-based study. The authors interpreted this to suggest that tests used to measure current cognitive abilities are more directly linked to the genetic risk factors of schizophrenia. In the present study, we also found that both measures of premorbid (NART) and current (composite score) cognitive abilities were not associated with $\mathrm{PGS}_{\mathrm{SCZ}}$ at all (and only nominally associated with the $\mathrm{PGS}_{\mathrm{COG}}$ in healthy controls). While some findings could be based on low statistical power, our study supports the notion that cognitive phenotypes differ in their associations with the genetic basis of schizophrenia.

Several previous studies have, however, found significant associations between $\mathrm{PGS}_{\mathrm{SCZ}}$ and cognitive phenotypes, while others have not ${ }^{20,22,23,60}$. In a recent review of PGS $\mathrm{SCZ}$ and cognitive functioning, the authors hypothesize that studies using improved cognitive phenotypes, such as the $\mathrm{MCCB}$, will detect associations ${ }^{29}$. In line with this, a recent study including 127 patients and 136 healthy controls found significant associations between $\mathrm{PGS}_{\mathrm{SCZ}}$ and all domains of the $\mathrm{MCCB}$, including the cognitive composite score ${ }^{19}$. The present study investigated several tests from the MCCB in a significantly larger sample. This suggests that refining the cognitive phenotypes alone will not increase the power to detect associations. Since our sample size is larger than most of the previous studies reporting significant associations, our lack of findings is also not primarily based on lack of statistical power relative to these studies.
Recent studies indicate a complex pattern of association between the genetic basis for cognition and schizophrenia: While most (but not all) schizophrenia risk alleles were associated with lower intelligence, around half of bipolar disorder risk alleles were associated with higher intelligence $^{61}$. Further, among the 75 loci shared between bipolar disorder and intelligence, there was an equal distribution of agonistic and antagonistic effect directions possibly explaining the overall non-significant correlation between these two phenotypes ${ }^{61}$. Another GWAS analysis reports a similar complex genetic relationship between schizophrenia and cognitive measures ${ }^{35}$. In line with this, other studies have found weak-and in some cases positive-associations between educational attainment as a proxy for cognitive abilities and schizophrenia ${ }^{62}$, despite behavioral data showing a strong negative association between schizophrenia and attaining higher education. Another study suggests that this may be caused by a positive association between educational attainment and risk alleles shared between schizophrenia and bipolar disorder, suppressing the negative association with risk alleles unique to schizophrenia ${ }^{63}$. Taken together, the findings indicate a highly complex and not uniformly negative association between schizophrenia risk variants and cognitive functioning that may explain the lack of association between $\mathrm{PGS}_{\mathrm{SCZ}}$ and cognitive abilities.

Underpowered GWAS discovery samples or imprecision in current methods used to generate $\mathrm{PGS}_{\mathrm{SCZ}}$ could account for lack of clear answers. The construction of the $\mathrm{PGS}_{\mathrm{SCZ}}$ is powered by design to explain variance in case-control status and the phenotypic variance explained by the currently available $\mathrm{PGS}_{\mathrm{SCZ}}$ is relatively ${ }_{\text {low }}{ }^{64}$. Here, increased sample sizes in the GWAS on which the PGS is based may generate PGSs with better predictive power and as the statistical power of GWASs increase, the improved PGS is may become a better tool for future personalized medicine ${ }^{65}$. As of now, any associations (or lack thereof) between PGSs and complex phenotypes should be interpreted with caution.

The finding regarding working memory is noteworthy. Working memory may be a particularly important domain because it has been suggested that it is at the core of the widespread deficits observed across psychotic disorders through its influence of proactive control ${ }^{66}$. According to theory, the inability to retain and organize information in short-term memory impairs the ability to proactively guide thoughts and behavior in an efficient manner and leads to observed impairments in several domains. A recent study of patients with psychotic disorders found significant associations between PGS for educational attainment and measures of working memory $^{60}$; however, with a relatively limited influence on the explained variance. The diagnostic composition, sample size, and cognitive measures were approximately the same 
as the current. Educational attainment is perceived as a less precise proxy or indication of cognitive abilities; the larger sample size of educational attainment GWASs could, however, lead to a better calculation of the educational attainment PGS. The study does not report directly on findings in their somewhat smaller healthy control sample. Taken together, our results support the notion that clinical and environmental factors in persons with a psychotic disorder could explain more variance in cognitive phenotypes than in healthy controls, and that current estimates may overstate the role of cognition as a part of the genetic etiology of psychotic disorders. Future studies should include a focus on working memory.

\section{Acknowledgements}

We thank every participant for their valuable contribution.

\section{Author details}

${ }^{1}$ NORMENT, Division of Mental Health and Addiction, Oslo University Hospital \& Institute of Clinical Medicine, University of Oslo, Oslo, Norway. ${ }^{2}$ Department of Psychology, Faculty of Social Sciences, University of Oslo, Oslo, Norway. ${ }^{3}$ Early Intervention in Psychosis Advisory Unit for South East Norway, Division of Mental Health and Addiction, Oslo University Hospital, Oslo, Norway. ${ }^{4}$ NORMENT, Department of Clinical Science, University of Bergen, Bergen, Norway. ${ }^{5}$ Department of Medical Genetics, Oslo University Hospital, Oslo, Norway

\section{Conflict of interest}

O.A.A. has received speaker's honorarium from Lundbeck and is a consultant for Healthlytix. For the remaining authors, there are no interests to declare.

\section{Publisher's note}

Springer Nature remains neutral with regard to jurisdictional claims in published maps and institutional affiliations.

Supplementary Information accompanies this paper at (https://doi.org/ 10.1038/s41398-020-01094-9).

Received: 31 December 2019 Revised: 4 October 2020 Accepted: 26 October 2020

Published online: 30 November 2020

\section{References}

1. Sheffield, J. M., Karcher, N. R. \& Barch, D. M. Cognitive deficits in psychotic disorders: a lifespan perspective. Neuropsychol. Rev. 28, 509-533 (2018).

2. Bowie, C. R. \& Harvey, P. D. Cognitive deficits and functional outcome in schizophrenia. Neuropsychiatr. Dis. Treat. 2, 531-536 (2006).

3. Hill, S. K. et al. Neuropsychological impairments in schizophrenia and psychotic bipolar disorder: findings from the Bipolar-Schizophrenia Network on Intermediate Phenotypes (B-SNIP) study. Am. J. Psychiatry 170, 1275-1284 (2013).

4. Mesholam-Gately, R. I., Giuliano, A. J., Goff, K. P., Faraone, S. V. \& Seidman, L. J. Neurocognition in first-episode schizophrenia: a meta-analytic review. Neuropsychology 23, 315-336 (2009).

5. Schaefer, J., Giangrande, E., Weinberger, D. R. \& Dickinson, D. The global cognitive impairment in schizophrenia: consistent over decades and around the world. Schizophr. Res. 150, 42-50 (2013).

6. Grande, I., Berk, M., Birmaher, B. \& Vieta, E. Bipolar disorder. Lancet 387 1561-1572 (2016).

7. Bora, E. \& Pantelis, C. Meta-analysis of cognitive impairment in first-episode bipolar disorder: comparison with first-episode schizophrenia and healthy controls. Schizophr. Bull. 41, 1095-1104 (2015).
8. Green, M. F. Cognitive impairment and functional outcome in schizophrenia and bipolar disorder. J. Clin. Psychiatry 67, 3-8 (2006).

9. Lichtenstein, P. et al. Common genetic determinants of schizophrenia and bipolar disorder in Swedish families: a population-based study. Lancet $\mathbf{3 7 3}$ 234-239 (2009).

10. Anttila, V. et al. Analysis of shared heritability in common disorders of the brain. Science 360, 1313 (2018).

11. Polderman, T. J. et al. Meta-analysis of the heritability of human traits based on fifty years of twin studies. Nat. Genet. 47, 702-709 (2015).

12. Hill, W. D., Harris, S. E. \& Deary, I. J. What genome-wide association studies reveal about the association between intelligence and mental health. Curr. Opin. Psychol. 27, 25-30 (2018).

13. Sitskoorn, M. M., Aleman, A., Ebisch, S. J., Appels, M. C. \& Kahn, R. S. Cognitive deficits in relatives of patients with schizophrenia: a meta-analysis. Schizophr. Res. 71, 285-295 (2004).

14. Glahn, D. C. et al. Neurocognitive endophenotypes for bipolar disorder identified in multiplex multigenerational families. Arch. Gen. Psychiatry 67, 168-177 (2010).

15. Toulopoulou, T. et al. Polygenic risk score increases schizophrenia liability through cognition-relevant pathways. Brain 142, 471-485 (2019).

16. Owen, M. J., Sawa, A. \& Mortensen, P. B. Schizophrenia. Lancet 388, 86-97 (2016).

17. Grande, I., Berk, M., Birmaher, B. \& Vieta, E. Bipolar disorder. Lancet 387, 1561-1572 (2016).

18. Schizophrenia Working Group of the Psychiatric Genomics, C. Biological insights from 108 schizophrenia-associated genetic loci. Nature 511, 421-427 (2014).

19. Nakahara, S. et al. Polygenic risk score, genome-wide association, and gene set analyses of cognitive domain deficits in schizophrenia. Schizophr. Res. 201, 393-399 (2018).

20. Xavier, R. M., Dungan, J. R., Keefe, R. S. E. \& Vorderstrasse, A. Polygenic signal for symptom dimensions and cognitive performance in patients with chronic schizophrenia. Schizophr. Res. Cogn. 12,11-19 (2018).

21. Whalley, H. C. et al. Dissection of major depressive disorder using polygenic risk scores for schizophrenia in two independent cohorts. Transl. Psychiatry 6 e938 (2016).

22. Walton, E. et al. Prefrontal inefficiency is associated with polygenic risk for schizophrenia. Schizophr. Bull. 40, 1263-1271 (2014).

23. Terwisscha van Scheltinga, A. F. et al. Genetic schizophrenia risk variants jointly modulate total brain and white matter volume. Biol. Psychiatry 73, 525-531 (2013).

24. Shafee, R. et al. Polygenic risk for schizophrenia and measured domains of cognition in individuals with psychosis and controls. Transl. Psychiatry $\mathbf{8}, 78$ (2018).

25. Richards, A. L. et al. The relationship between polygenic risk scores and cognition in schizophrenia. Schizophr. Bull. 46, 336-344 (2020).

26. Bouchard, T. J. Jr. Genetic influence on human intelligence (Spearman's g): how much? Ann. Hum. Biol. 36, 527-544 (2009).

27. Panizzon, M. S. et al. Genetic and environmental influences of general cognitive ability: is g a valid latent construct? Intelligence $\mathbf{4 3}$, 65-76 (2014).

28. Haworth, C. M. et al. The heritability of general cognitive ability increases linearly from childhood to young adulthood. Mol. Psychiatry 15, 1112-1120 (2010).

29. Schaupp, S. K., Schulze, T. G. \& Budde, M. Let's talk about the association between schizophrenia polygenic risk scores and cognition in patients and the general population: a review. J. Psychiatry Brain Sci. 3, 12 (2018).

30. Hubbard, L. et al. Evidence of common genetic overlap between schizophrenia and cognition. Schizophr. Bull. 42, 832-842 (2016).

31. Lencz, T. et al. Molecular genetic evidence for overlap between general cognitive ability and risk for schizophrenia: a report from the Cognitive Genomics consorTium (COGENT). Mol. Psychiatry 19, 168-174 (2014).

32. Toulopoulou, T. et al. Substantial genetic overlap between neurocognition and schizophrenia: genetic modeling in twin samples. Arch. Gen. Psychiatry 64, 1348-1355 (2007).

33. Toulopoulou, T. et al. Impaired intellect and memory: a missing link between genetic risk and schizophrenia? Arch. Gen. Psychiatry 67, 905-913 (2010).

34. Fowler, T., Zammit, S., Owen, M. J. \& Rasmussen, F. A population-based study of shared genetic variation between premorbid IQ and psychosis among male twin pairs and sibling pairs from Sweden. Arch. Gen. Psychiatry 69, 460-466 (2012) 
35. Davies, G. et al. Genome-wide association study of cognitive functions and educational attainment in UK Biobank ( $N=112$ 151). Mol. Psychiatry 21, 758-767 (2016).

36. American Psychiatric Association. DSM-IV-TR: diagnostic and statistical manual of mental disorders, text revision. Am. Psychiatr. Assoc. 75, 78-85 (2000).

37. Spitzer, R. L., Kroenke, K. \& Williams, J. B., Group, P. H. Q. P. C. S. Validation and utility of a self-report version of PRIME-MD: the PHQ primary care study. JAMA 282, 1737-1744 (1999).

38. Kay, S. R., Fiszbein, A. \& Opler, L. A. The positive and negative syndrome scale (PANSS) for schizophrenia. Schizophr. Bull. 13, 261-276 (1987).

39. Wallwork, R. S., Fortgang, R., Hashimoto, R., Weinberger, D. R. \& Dickinson, D. Searching for a consensus five-factor model of the Positive and Negative Syndrome Scale for schizophrenia. Schizophr. Res. 137, 246-250 (2012).

40. Hildebrand, M. \& Noteborn, M. G. Exploration of the (interrater) reliability and latent factor structure of the Alcohol Use Disorders Identification Test (AUDIT) and the Drug Use Disorders Identification Test (DUDIT) in a sample of Dutch probationers. Subst. Use Misuse 50, 1294-1306 (2015).

41. Leucht, S., Samara, M., Heres, S. \& Davis, J. M. Dose Equivalents for Antipsychotic Drugs: the DDD Method. Schizophr. Bull. 42(Suppl. 1), S90-S94 (2016).

42. Purcell, S. et al. PLINK: a tool set for whole-genome association and population-based linkage analyses. Am. J. Hum. Genet. 81, 559-575 (2007).

43. Loh, P. R. et al. Reference-based phasing using the Haplotype Reference Consortium panel. Nat. Genet. 48, 1443-1448 (2016).

44. Li, Y., Willer, C. J., Ding, J., Scheet, P. \& Abecasis, G. R. MaCH: using sequence and genotype data to estimate haplotypes and unobserved genotypes. Genet. Epidemiol. 34, 816-834 (2010).

45. Das, $\mathrm{S}$. et al. Next-generation genotype imputation service and methods. Nat. Genet. 48, 1284-1287 (2016).

46. McCarthy, S. et al. A reference panel of 64,976 haplotypes for genotype imputation. Nat. Genet. 48, 1279-1283 (2016).

47. Purcell, S. M. et al. Common polygenic variation contributes to risk of schizophrenia and bipolar disorder. Nature 460, 748-752 (2009).

48. Choi, S. W. \& O'Reilly, P. F. PRSice-2: Polygenic Risk Score software for biobankscale data. Gigascience 8, https://doi.org/10.1093/gigascience/giz082 (2019).

49. Trampush, J. W. et al. GWAS meta-analysis reveals novel loci and genetic correlates for general cognitive function: a report from the COGENT consortium. Mol. Psychiatry 22, 1651-1652 (2017).

50. Nuechterlein, K. H. et al. The MATRICS Consensus Cognitive Battery, part 1: test selection, reliability, and validity. Am. J. Psychiatry 165, 203-213 (2008).
51. Nerhus, M. et al. Vitamin D deficiency associated with cognitive functioning in psychotic disorders. J. Clin. Psychiatry 78, e750-e757 (2017).

52. Bortolato, B., Miskowiak, K. W., Kohler, C. A., Vieta, E. \& Carvalho, A. F. Cognitive dysfunction in bipolar disorder and schizophrenia: a systematic review of meta-analyses. Neuropsychiatr. Dis. Treat. 11, 3111-3125 (2015).

53. Delis, D. C., Kaplan, E. \& Kramer, J. H. Delis-Kaplan Executive Function System (DKEFS) (Psychological Corporation, San Antonio, 2001).

54. Wechsler, D. Wechsler Adult Intelligence Scale-3rd Edition (WAIS-III) Manual (Psychological Corporation, New York, 1997).

55. Keefe, R. S. et al. The Brief Assessment of Cognition in Schizophrenia: reliability, sensitivity, and comparison with a standard neurocognitive battery. Schizophr. Res. 68, 283-297 (2004).

56. Benedict, R. H. B., Schretlen, D., Groninger, L. \& Brandt, J. Hopkins Verbal Learning Test Revised: normative data and analysis of inter-form and testretest reliability. Clin. Neuropsychologist 12, 43-55 (1998).

57. Blair, J. R. \& Spreen, O. Predicting premorbid IQ: a revision of the National Adult Reading Test. Clin. Neuropsychologist 3, 129-136 (1989).

58. Vaskinn, A. et al. Cognitive heterogeneity across schizophrenia and bipolar disorder: a cluster analysis of intellectual trajectories. J. Int. Neuropsychol. Soc. https://doi.org/10.1017/s1355617720000442, 1-13 (2020).

59. Weaver, B. \& Wuensch, K. L. SPSS and SAS programs for comparing Pearson correlations and OLS regression coefficients. Behav. Res. Methods 45, 880-895 (2013).

60. Comes, A. L. et al. The genetic relationship between educational attainment and cognitive performance in major psychiatric disorders. Transl. Psychiatry $\mathbf{9}$, 210 (2019).

61. Smeland, O. B. et al. Genome-wide analysis reveals extensive genetic overlap between schizophrenia, bipolar disorder, and intelligence. Mol. Psychiatry https://doi.org/10.1038/s41380-018-0332-x (2019).

62. Okbay, A. et al. Genome-wide association study identifies 74 loci associated with educational attainment. Nature 533, 539-542 (2016).

63. Bansal, V. et al. Genome-wide association study results for educational attainment aid in identifying genetic heterogeneity of schizophrenia. Nat. Commun. 9, 3078 (2018).

64. Lee, S. H., Goddard, M. E., Wray, N. R. \& Visscher, P. M. A better coefficient of determination for genetic profile analysis. Genet. Epidemiol. 36, 214-224 (2012).

65. Torkamani, A., Wineinger, N. E. \& Topol, E. J. The personal and clinical utility of polygenic risk scores. Nat. Res. Genet. 1, 581-590 (2018).

66. Barch, D. M. \& Sheffield, J. M. Cognitive impairments in psychotic disorders: common mechanisms and measurement. World Psychiatry 13, 224-232 (2014). 